DOI: https://doi.org/10.24297/jap.v16i1.8237

\title{
Study on MHD Cylindrical Couette Flow and Rheological Properties of some Magnetic Suspensions
}

\author{
S.E.E. Hamza
}

Physics Department, Faculty of Science, Benha University, Benha, Egypt

salah.hamza@fsc.bu.edu.eg

\begin{abstract}
The study of magnetic suspensions (MS) and magnetic field effects on their rheological properties is of evident practical importance due to its ability to orient and change their physical properties, especially their viscosity, by magnetic fields. This research presents the effect of a uniform magnetic field on the flow of MS in the annular region between two concentric cylinders. The motion of the fluid is due to the rotation of the inner cylinder with a constant angular velocity. An exact solution of the governing equations is obtained in the form of modified Bessel functions of the first and second kinds. The torque, which must be applied to the inner cylinder in order to maintain the rotation, is also calculated. The results show that as the magnetic parameter increases, the velocity profile decreases, while the torque increases due to the effect of magnetic force against the flow direction.

In order to model the magnetoviscous effects, experiments were performed for different shear rates and different magnetic field strengths by using specially designed rheometers. The studied samples are iron oxidewater-glycerol system, $\mathrm{Fe}_{3} \mathrm{O}_{4}$ ferrofluid nanoparticles, MAG DX biocompatible ferrofluid. The theoretical analysis is based on Giesekus model for MS. This model gives more accurate results and takes into account the effects of viscoelastic shear thinning characteristics. It is found that a magnetic field increases the viscosity of all suspensions under consideration. Finally, new proposed correlation for the viscosity of MS as a function of both shear rate and magnetic field has been suggested.
\end{abstract}

\section{Indexing terms/Keywords}

MHD flow, Concentric cylinders, Couette flow, Bessel function, Magnetic suspensions, Ferrofluids, Giesekus model, Nano-particles.

\section{Academic Discipline And Sub-Disciplines}

Physics, Fluid Mechanics

\section{SUBJECT CLASSIFICATION}

Rheology of Polymers

\section{TYPE (METHOD/APPROACH)}

Theoretical Study.

\section{INTRODUCTION}

Couette flow is one in which the shearing surfaces are coaxial cylinders which rotate with an individual angular velocities around their common axis. An analytical solution to the flow of viscous Newtonian fluid through vertical annulus can be found in the classical textbooks of Bird et al. [1]. In real life, many fluids exhibit nonNewtonian nature. The study of the flow of such fluids is necessary due to its numerous technical and 
industrial applications such as petroleum, oil refining, food industries etc. A single fluid model cannot describe the behavior of all non-Newtonian fluids. Therefore, several models have been proposed. One of these models is the Oldroyd-B fluid model. This fluid has been used quite widely in many applications and the results of simulations fit experimental data in a wide range.

One of the most important features of MS is the possibility to change their physical properties, especially their viscosities, by means of moderate magnetic fields. This capability makes MS very useful in the fields of engineering, medicine and fundamental researches. Under certain conditions, experimental results correlate the change of the viscosity of sheared MS under the influence of a magnetic field to the internal structure formation. Because of its specific characteristics, MS behaves as functional fluid and has found many useful applications such as dynamic sealing, dampers, heat conductors, temperature and magnetic field sensors, DNA detection, magnetic drug targeting and cancer hyperthermia treatment [2-4].

Magnetic fluids are a smart materials which are prepared by dispersing the magnetic particles into the carrying fluids, either aqueous or non-aqueous. Because of the magnetic dipolar-dipolar interaction among the magnetic particles, the magnetic fluids possessed typical magnetoviscous effects [5]. Experimental investigations have shown that an increase of the magnetic field strength yields an increase of the fluid's viscosity, the so-called magnetoviscous effect [6]. In the classical theory of rotational viscosity, this behavior is explained by the hindrance of the free rotation of particles in a shear flow by magnetic fields. This theory applies for highly diluted ferrofluids, assuming vanishing interactions between the particles. However, the commercial ferrofluids used for most applications are generally concentrated and, therefore, the interaction between the particles cannot be ignored [7].

Magnetic suspensions can be considered as liquid mixtures made of magnetic particles chains and small molecules of solvent. At rest, the chains of suspensions are randomly entangled and they do not set up the suspensions structure. When the fluid is in motion, the chains tend to align themselves parallel to the direction of flow. This tendency increases with increasing shear rate, so that the effective viscosity decreases [8]. In dilute suspensions, the rheology of suspension depends solely on the dynamics of an individual chain and the number of chains in the system. At high concentrations, interactions between suspension's particles impact the rheology in a significant way. As concentration increases, suspensions exhibit a change from fluid-like to more elastic-like behavior [9]. Viscosities of MS are known to be variable quantities which decrease with increasing shear rate. This shear thinning effect has been known for a long time, but its adequate explanation is still absent. In the case of concentrated suspensions, a varying viscosity is assumed to be due to the entanglement of magnetic particles chains. For a dilute solution, viscosity variations are associated with a behavior of separate particles in the flow, i.e., their deformation, orientation, etc.

The orientation of magnetic particles in solvent under the influence of an external magnetic field is of great importance owing to the possibility of changes in structure and the products formation. Under the effect of magnetic field these particles may rearrange themselves taking the same direction as the magnetic field lines (i.e. oriented parallel to the magnetic lines), a circumstance that lead to an increase in suspension viscosity. The effects of shear rate, magnetic field and concentration on the rheological properties of MS were investigated by Vshivkov et al. [10], Ghasemi et al. [11] and Hong et al. [12]. Many researchers have tried to explain the magnetoviscous effect $[13,14]$. Although the theories of chains have provided good explanations for the magnetoviscous effect, there are still many unexplained matters in this area. It is widely accepted that magnetoviscous effect is due to the complicity of the interaction originates from the disorder to order transition of the particles under applying an external magnetic field [15]. Therefore, the magnetic property, shape, size, and inner structure of the magnetic particles become the critical roles for affecting the rheological properties $[16,17]$. During the past decade, several groups performed intensive work in this area to investigate the magnetoviscous mechanism [18-23].

A ferrofluid is a suspension that consists of ferromagnetic nanoparticles dispersed in a carrier liquid such as water or organic solvents. Usually solid particles in these fluids are about $10 \mathrm{~nm}$ in size. In most engineering 
applications of ferrofluids, the magnetic material can be one of $\mathrm{XFe}_{2} \mathrm{O}_{4}(\mathrm{X}=\mathrm{Fe}, \mathrm{Co}, \mathrm{Cu}, \mathrm{Ni}, \mathrm{Zn}, \mathrm{Mg}, \mathrm{Ba})$ ferrites. The most commonly used ferrite is magnetite : $\left.\mathrm{Fe}_{3} \mathrm{O}_{4}\right)$. Hence, most studies have focused on the effects of magnetic field on the rheological properties of this ferrite [24]. A special character of ferrofluids is that, their flow and properties can be controlled by applying moderate magnetic fields. This has been a source of various technical and clinical applications $[25,26]$. Such fluids show non-Newtonian behaviour in presence of magnetic field. In different applications, the key parameters that dominate the process performance are viscosity and thermal conductivity of the ferrofluid. Recently, $\mathrm{Fe}_{3} \mathrm{O}_{4}$ based magnetic fluids have attracted increasing interests since their better stability than the common carbonyl iron based magnetorheological fluid [27-29].

At present, the flow field of a viscoelastic fluid in the annular region between two concentric cylinders is investigated. The inner cylinder rotates with a uniform angular velocity about its own z-axis centered in the origin of the system and the outer cylinder is being at rest. To benefit from this theoretical study in the interpretation of magnetoviscous phenomenon, we reconsider the experimental data measured by Vshivkov et al. [27], Dhumal et al. [28], Nowak et al. [29] and Linke et al. [30] for iron oxide and biocompatible ferrofluids. We collect and reorder their experimental data and carry out its theoretical analysis based on Giesekus model for viscoelastic suspensions. As we have seen from our previous papers [31-33] this model takes into account the effects of shear thinning characteristics on the viscosity of the polymer solutions and gives more accurate results. In addition, the effects of magnetic field on the magnetorheological properties of the used MS are investigated. The chosen model fits adequately the experimental data.

\section{GOVERNING EQUATIONS}

To get a comprehensive idea about the behavior of a viscoelastic fluid, we consider an incompressible Oldroyd-B fluid for which the Cauchy stress tensor $\underline{\underline{T}}$ is given as follows:

$$
\begin{aligned}
& \underline{\underline{T}}=-\mathrm{pl}+\underline{\underline{s}}, \\
& \stackrel{\underline{s}}{=}+\lambda_{1} \underline{\underline{s}}=2 \mu\left(\underline{\underline{\gamma}}+\lambda_{2} \underset{\underline{\gamma}}{=}\right),
\end{aligned}
$$

where $\mathrm{p}$ is the pressure, $\underline{\underline{s}}$ is the extra stress tensor, $\underset{\underline{\gamma}}{\dot{\gamma}}$ is the rate of deformation tensor, $\mu$ is the shear viscosity while $\lambda_{1}, \lambda_{2}$ are the relaxation and retardation times respectively and $\underset{\underline{\gamma}}{\stackrel{\nabla}{=}}$ is the upper convected derivative of $\underset{\underline{\dot{\gamma}}}{=}$ :

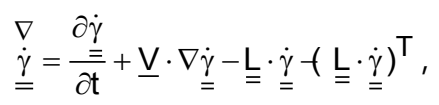

where $\underline{\mathrm{V}}$ is the velocity vector. The tensors $\underline{\underline{\underline{L}}}$ and $\underset{\underline{\dot{\gamma}}}{\underline{a}}$ are defined as:

$$
\left.\underline{\underline{L}}=\nabla \underline{\mathrm{V}}, \quad \underline{\underline{\gamma}}=\frac{1}{2} \underline{\underline{L}}+\underline{\underline{L}}^{\top}\right) .
$$

Let us consider the motion of a viscoelastic fluid between two semi infinite concentric cylinders of circular cross sections having radii $R_{1}$ and $R_{2}\left(R_{2}>R_{1}\right)$. The inner cylinder rotating steadily around its axis by an angular velocity $\Omega$ while the outer cylinder is kept at rest. The fluid is assumed to be an incompressible and electrically conducting of constant density $\rho$. The Cauchy dynamical equation of motion and the equation of continuity are given by: 


$$
\rho \underline{\mathrm{V}} \cdot \nabla \underline{\mathrm{V}}=\nabla \cdot \underline{\underline{T}}+\underline{\mathrm{f}},
$$

and

$$
\nabla \cdot \underline{V}=0,
$$

where $f$ is the body force per unit volume. The presence of a magnetic field requires that an additional force be included in the equations of fluid motion aside from the usual pressure and shear forces. The added force takes the form:

$$
\underline{\mathrm{f}}=\underline{\mathrm{J}} \times \underline{\mathrm{B}},
$$

where $\mathrm{J}$ is the current density and $\underline{B}$ is the magnetic induction vector. The current density is given as:

$$
\underline{\mathrm{J}}=\sigma(\underline{\mathrm{E}}+\underline{\mathrm{V}} \times \underline{\mathrm{B}}),
$$

in which the terms $\sigma \underline{E}$ and $\sigma(\underline{V} \times \underline{B})$, respectively, represent the conduction and induction currents and $\sigma$ is the electrical conductivity.

We shall make use of the cylindrical coordinate system $(r, \theta, z)$ for the solution of this boundary value problem. The $z$-axis is coincident with the two cylinders axis and $\theta>0$ anticlockwise, see figure 1 . We shall seek an axisymmetric two dimensional solution and this assume that the only nonzero component of velocity is then $v(r)$

$$
\underline{v}=[0, v(r), 0] \text {. }
$$

Furthermore, this velocity component as well as the stress components and the pressure will be functions of $r$ only.

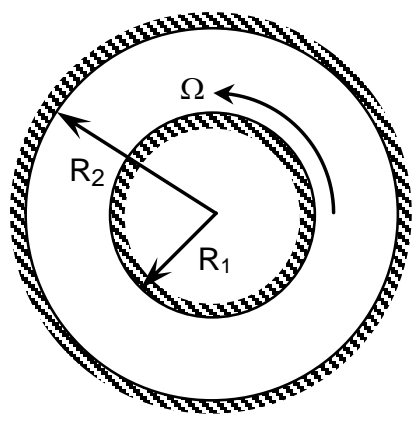

(a)

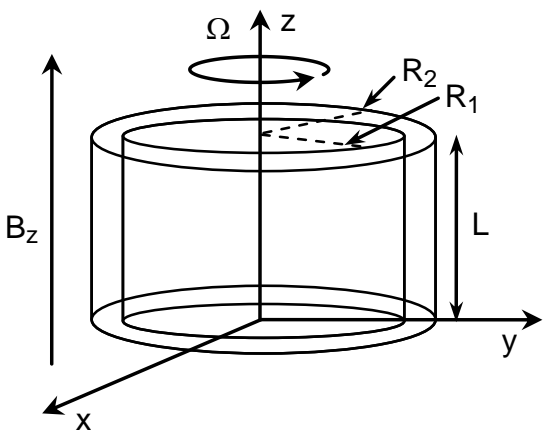

(b)

Fig 1: Geometry of the problem: (a) cross-section of the cylinders and (b) schematic representation of viscosity determination with lines of force directed parallel to the rotation axis of the rotor.

In the present study, we assume that the magnetic field is in the direction of z-axis. Neglecting the electric field $\underline{E}$ in equation 8 and replacing $\underline{B}$ by the externally applied field

$$
\underline{B}=B_{O} \hat{z},
$$


with the choice of the velocity given in equation 9 , the continuity equation is satisfied automatically and Lorentz force takes the form:

$$
f_{\theta}=-\sigma B_{o}^{2} v,
$$

hence, the $r$ - and $\theta$-components of the equation of motion are written, respectively, as:

$$
\begin{aligned}
& \frac{s_{\theta \theta}}{r}+\frac{d p}{d r}=\rho \frac{v^{2}}{r}, \\
& \frac{2 s_{r \theta}}{r}+\frac{d s_{r \theta}}{d r}-\sigma B_{O}^{2} v=0 .
\end{aligned}
$$

The constitutive equation, equation 1 , gives:

$$
\begin{aligned}
& \mathrm{s}_{\mathrm{rr}}=0, \\
& \mathrm{~s}_{\mathrm{r} \theta}=\mu\left(\frac{\mathrm{d} v}{\mathrm{dr}}-\frac{\mathrm{v}}{\mathrm{r}}\right), \\
& \mathrm{s}_{\theta \theta}=2 \mu\left(\lambda_{1}-\lambda_{2}\right)\left(\frac{\mathrm{du}}{\mathrm{dr}}-\frac{\mathrm{v}}{\mathrm{r}}\right)^{2} .
\end{aligned}
$$

Equations 12 and 13 represent the governing flow equations with the boundary conditions:

$$
v\left(R_{1}\right)=R_{1} \Omega \text {, and } v\left(R_{2}\right)=0 \text {. }
$$

Introducing the following change of variables:

$$
\stackrel{*}{r}=\frac{r}{R_{1}}, \stackrel{*}{v}=\frac{v}{R_{1} \Omega}, \stackrel{*}{p}=\frac{p}{\rho\left(R_{1} \Omega\right)^{2}}, \stackrel{*}{s_{i j}}=\frac{s_{\bar{I}}}{\rho\left(R_{1} \Omega\right)^{2}}, \quad \stackrel{*}{\lambda_{1}}=\frac{\mu \dot{\lambda}_{1}}{\rho R_{1}^{2}}, \quad \stackrel{*}{\lambda_{2}}=\frac{\mu \hat{i}_{2}}{\rho R_{1}^{2}},
$$

and defining the dimensionless constants

$$
\mathrm{Re}=\frac{\Omega \mathrm{R}_{1}^{2}}{v}, \quad \mathrm{~m}=\frac{\sigma \mathrm{B}_{\mathrm{o}}^{2} \mathrm{R}^{2}}{\mu}, \quad \beta=\frac{\mathrm{R}_{2}}{\mathrm{R}_{1}}, \quad \Delta \stackrel{*}{\lambda}=\stackrel{*}{\lambda_{1}}-\lambda_{2}^{*},
$$

with $v=\mu / \rho$ is the kinematic viscosity. After substitution from equations 15 into equations 12 and 13 we get (dropping the dimensionless mark "*" for simplicity):

$$
\begin{aligned}
& \mathrm{s}_{\mathrm{r} \theta}=\frac{1}{\operatorname{Re}}\left(\frac{\mathrm{du}}{\mathrm{dr}}-\frac{\mathrm{v}}{\mathrm{r}}\right), \\
& \mathrm{s}_{\theta \theta}=2 \Delta \lambda\left(\frac{\mathrm{du}}{\mathrm{dr}}-\frac{\mathrm{v}}{\mathrm{r}}\right)^{2} . \\
& \frac{\mathrm{s}_{\theta \theta}}{\mathrm{r}}+\frac{\mathrm{dp}}{\mathrm{dr}}=\frac{\mathrm{v}^{2}}{\mathrm{r}},
\end{aligned}
$$




$$
\frac{2 s_{r \theta}}{r}+\frac{d \tau_{r \theta}}{d r}-\frac{\sigma B_{o}^{2}}{\rho \Omega} v=0 .
$$

Substituting from equation $16 \mathrm{~b}$ for $\mathrm{s}_{\theta \theta}$ into equation $16 \mathrm{c}$ gives the pressure gradient,

$$
\frac{d p}{d r}=\frac{v^{2}}{r}-\frac{2 \Delta \lambda}{r}\left(\frac{d v}{d r}-\frac{v}{r}\right)^{2},
$$

using equations $16 \mathrm{a}$ and $16 \mathrm{~d}$, it is easy to prove that the velocity component $v$ satisfies the second-order boundary value problem:

$$
\frac{d^{2} v}{d r^{2}}+\frac{1}{r} \frac{d v}{d r}-\left(m^{2}+\frac{1}{r^{2}}\right) v=0
$$

with the boundary conditions:

$$
v(1)=1, \quad \text { and } \quad v(\beta)=0 .
$$

Equations 18 and 19 admit the solution:

$$
v(r)=C_{1} K_{1}(m r)+C_{2} l_{1}(m r),
$$

with

$$
C_{1}=\frac{l_{1}(m \beta)}{l_{1}(m \beta) K_{1}(m)-l_{1}(m) K_{1}(m \beta)},
$$

and

$$
C_{2}=\frac{-K_{1}(m \beta)}{l_{1}(m \beta) K_{1}(m)-l_{1}(m) K_{1}(m \beta)} .
$$

The functions $(f(x)$ and $K(x)$ are the modified Bessel functions of the first and second kind respectively. The symbol $m$ represents the magnetic parameter. The magnitude of $m$ is an index to the relative importance of magnetic forces. For $m=0$, the magnetic forces are absent. Increasing $m$, the magnetic forces become important.

Substituting $(r)$ and its derivatives from equation 20 into equations $16 \mathrm{a}$ and $16 \mathrm{~b}$, the shear and normal stresses $s_{r \theta}$ and $s_{\theta \theta}$ take the final forms:

$$
\begin{aligned}
& s_{r \theta}=\frac{-m}{\operatorname{Re}}\left[c_{1} K_{2}(m r)-C_{2} l_{2}(m r)\right], \\
& s_{\theta \theta}=-2 m^{2} \Delta z\left[C_{1} K_{2}(m r)-C_{2} l_{2}(m r)\right]^{2},
\end{aligned}
$$

also the dimensionless torque, which must be applied in order to maintain the rotation, is calculated as:

$$
\mathrm{T}=2 \pi \mathrm{r}^{2} \mathrm{~S}_{\mathrm{r} \theta} .
$$


The torques on the inner cylinder $(r=1)$ and outer cylinder $(r=\beta)$ are given as:

$$
\begin{aligned}
& \left.\tau\right|_{r=1}=\frac{-2 \pi m}{\operatorname{Re}}\left[C_{1} K_{2}(m)-C_{2} I_{2}(m)\right] \\
& \left.\tau\right|_{r=\beta}=\frac{-2 \pi \beta^{2} m}{\operatorname{Re}}\left[C_{1} K_{2}(m \beta)-C_{2} I_{2}(m \beta)\right] .
\end{aligned}
$$

Notice that, the velocity field $\varphi r$ ) and the shear stress $s_{r \theta}$ depend only on the geometrical parameters, but not on $\lambda_{1}$ or $\lambda_{2}$ which occurs in Newtonian case. Only the normal stress $s_{\theta \theta}$ depends on $\lambda_{1}$ and $\lambda_{2}$.

The variation of velocity field for $1 \leq r \leq \beta$ is calculated from equation 20 for different values of the included physical parameters and is shown in figure $2 a$ for fixed $\mathrm{m}$. The influence of magnetic parameter $\mathrm{m}$ on the velocity profile is presented in figure $2 b$ for fixed $\beta$. From these figures, it is noted that an increase in magnetic parameter $m$ reduces the velocity profile monotonically due to the effect of the magnetic force against the direction of the flow.

The increasing of the magnetic parameter m produces a variation in the whole flow and, as a consequence, in the shear stress all over. The later becomes larger for large $m$, as expected. The same phenomenon is shown in the dimensionless torque, figures. $3 a$ and $3 b$.
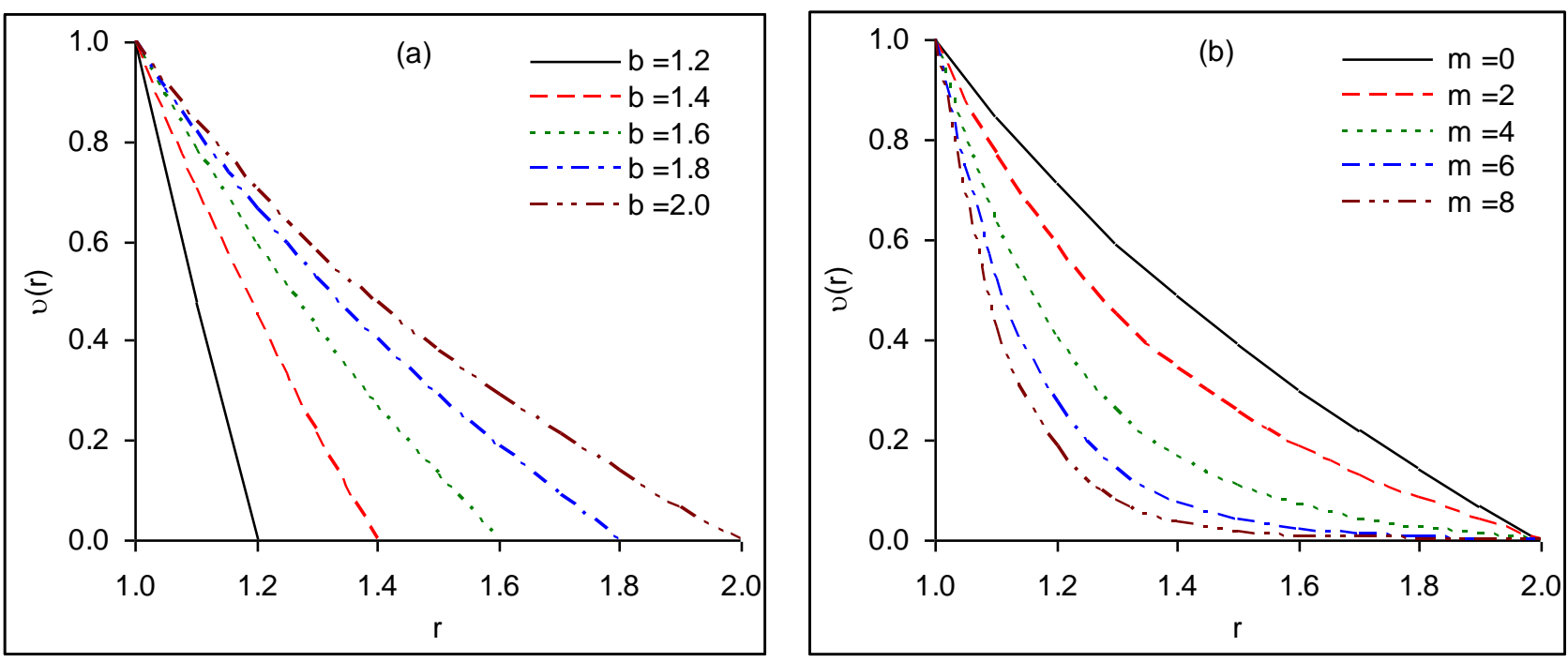

Fig 2: (a) The velocity field at $m=0.5$ and (b) The effect of magnetic field on $\psi r$ ) at $\beta=2$. 

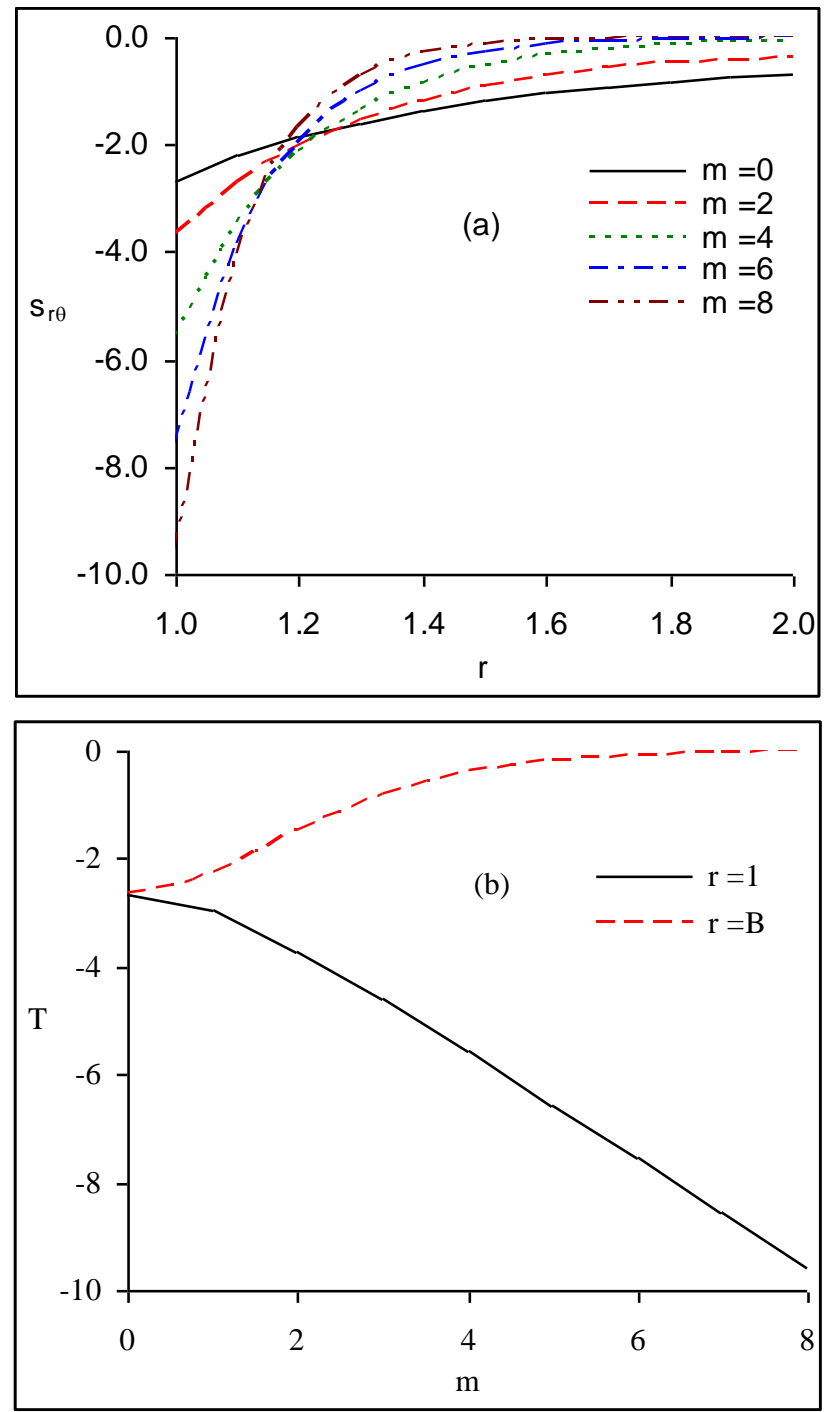

Fig 3: (a) The shear stress at $\beta=2$ and (b) The torque on the outer and inner cylinder surfaces.

From the theoretical solution and the obtained curves, we can conclude the following remarks:

- Increasing the magnetic parameter $m$, the velocity profile reduces monotonically due to the effect of magnetic force against the flow direction.

- $\quad$ The shear stress and torque become larger for large magnetic parameter $\mathrm{m}$.

- $\quad$ An increasing in $\beta$ yields an effect opposite to that of the magnetic field.

\section{TEST MATERIALS AND EXPERIMENTAL MEASUREMENTS}

Since the viscosity of MS is very important factor, it is necessary to collect some of the viscometric data to get more precise formula, which describe the viscosity. In this work, four different MS were studied. Vshivkov et al. [27] employed a suspension of iron oxide $\left(\mathrm{Fe}_{2.8} \mathrm{O}_{4}\right)$ nanoparticles in glycerol-water solution. Iron oxide nanopowder colored black with average particle diameter of $22 \mathrm{~nm}$ was used. The viscosity of the suspension was measured using a modified Rheotest RN 4.1 rheometer a coaxial cylindrical work unit made of a weak magnetic material (brass). A magnetic field was generated using a equipped with magnetic system generating a permanent magnetic field with strength of $280 \mathrm{kA} / \mathrm{m}$ and field lines directed parallel to the rotor-rotation 
axis. Vshivkov and his group investigated experimentally the changes in the viscosity of iron oxide over shear rates $0 \leq \dot{\gamma} \leq 30 \mathrm{~s}^{-1}$. Figure 4 shows schematic representation of Rheotest RN 4.1 rheometer and its installation method in magnetic field. The working unit was exposed to a magnetic field and the viscosity was measured while shear rate was gradually increased from 0 to $30 \mathrm{~s}^{-1}$. Table 1 presents the rheological data for $\mathrm{Fe}_{2.8} \mathrm{O}_{4}$ suspension for different magnetic flux density.
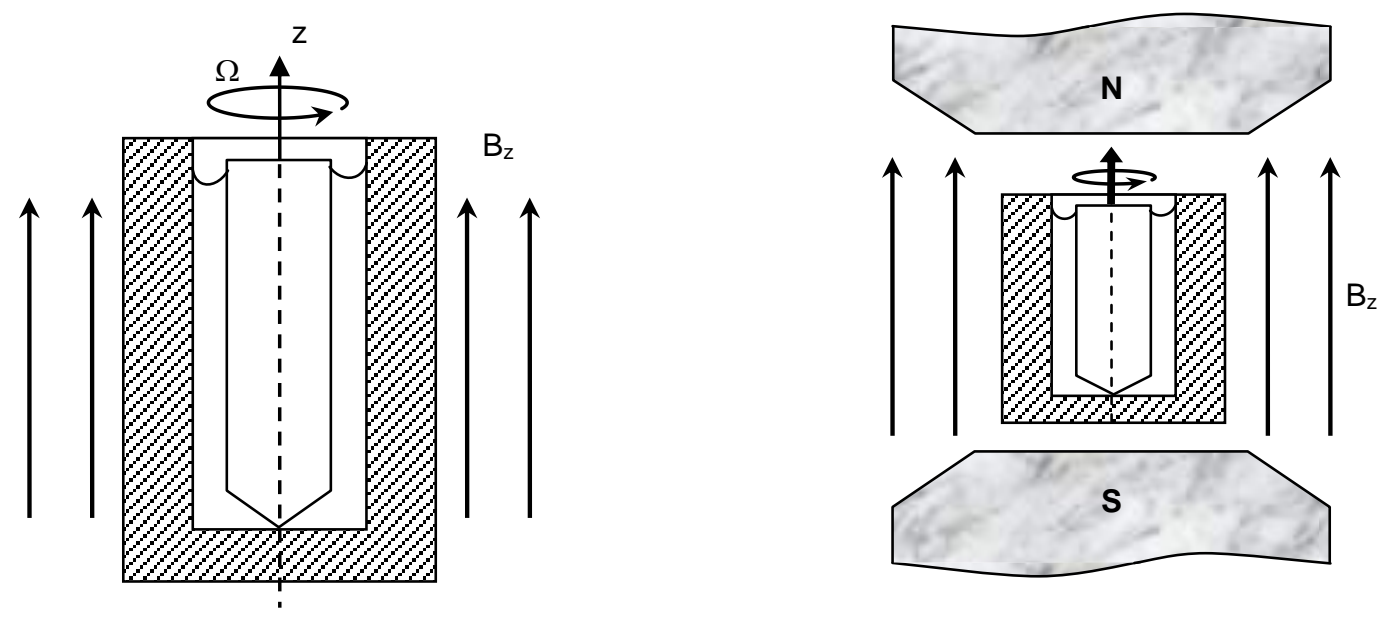

Fig 4: Schematic representation for installation method of the rheometer in magnetic field (side view), where $\mathrm{N}$ and $\mathrm{S}$ are electromagnet poles $\mathrm{B}_{\mathrm{z}}$ are the magnetic field intensity in z-direction.

Table 1. $\mathrm{Fe}_{2.8} \mathrm{O}_{4}$ ferrofluid viscosity at different magnetic flux density, Vshivkov et al. [28]

\begin{tabular}{|c|c|c|c|}
\hline \multirow{2}{*}{ Shear rate $\left.\chi^{-1} \mathrm{~s}^{-1}\right)$} & \multicolumn{3}{|c|}{ Experimental viscosity $\eta(\mathrm{Pa} \cdot \mathrm{s})$} \\
\cline { 2 - 4 } & $\mathrm{B}_{\mathrm{O}}=0.0 \mathrm{kA} / \mathrm{m}$ & $\mathrm{B}_{\mathrm{O}}=280 \mathrm{kA} / \mathrm{m}$ & $\mathrm{B}_{\mathrm{O}}=290 \mathrm{kA} / \mathrm{m}$ \\
\hline 2 & --- & --- & 3.53 \\
\hline 5 & 0.29 & 0.90 & 2.00 \\
\hline 10 & 0.18 & 0.60 & 1.21 \\
\hline 15 & 0.12 & 0.45 & 0.90 \\
\hline 20 & 0.12 & 0.41 & 0.80 \\
\hline 25 & 0.12 & 0.37 & 0.71 \\
\hline 30 & 0.12 & 0.34 & 0.65 \\
\hline
\end{tabular}

The materials and preparation of $\mathrm{Fe}_{3} \mathrm{O}_{4}$ ferrofluid nanoparticles were employed by Dhumal et al. [28]. The rheological measurements were carried out using MCR300 rheometer, see table 2. A special plate-plate spindle, TG16-MRD, was used for all measurements. A coaxial magnetic field in perpendicular direction to the sample was applied during the measurement. The rheometer is equipped with a constant temperature 
thermostatic bath unit that gives a very good temperature control over an extended time. Dhumal et al. used five different magnetic flux densities which are ranged from OT to 0.4T (with 0.1T increments). In all experimental range, the shear rate was varied from $0 \mathrm{~s}^{-1}$ to $100 \mathrm{~s}^{-1}$, see table 2 .

Table 2. $\mathrm{Fe}_{3} \mathrm{O}_{4}$ ferrofluid viscosity at different magnetic flux density, Dhumal et al. [28].

\begin{tabular}{|c|c|c|c|c|c|}
\hline \multirow{2}{*}{ Shear rate $\left.\dot{x} \mathrm{~s}^{-1}\right)$} & \multicolumn{5}{|c|}{ Experimental viscosity $\eta(\mathrm{mPa} \cdot \mathrm{s})$} \\
\hline & $\mathrm{B}_{\mathrm{O}}=0 \mathrm{~T}$ & $\mathrm{~B}_{\mathrm{O}}=0.1 \mathrm{~T}$ & $\mathrm{~B}_{\mathrm{O}}=0.2 \mathrm{~T}$ & $\mathrm{~B}_{\mathrm{O}}=0.3 \mathrm{~T}$ & $\mathrm{~B}_{\mathrm{O}}=0.4 \mathrm{~T}$ \\
\hline 3 & 60.34 & 260.00 & 340.45 & 400.82 & 469.96 \\
\hline 7 & 30.27 & 162.79 & 208.97 & 261.18 & 305.37 \\
\hline 10 & 22.29 & 128.72 & 174.91 & 207.03 & 237.15 \\
\hline 13 & 20.32 & 100.00 & 142.85 & 170.93 & 201.07 \\
\hline 17 & 16.41 & 88.70 & 122.83 & 144.92 & 173.03 \\
\hline 20 & 14.45 & 80.73 & 112.86 & 130.93 & 153.02 \\
\hline 23 & 12.52 & 76.78 & 104.89 & 124.97 & 139.04 \\
\hline 27 & 12.58 & 68.82 & 94.92 & 112.99 & 129.06 \\
\hline 30 & 12.65 & 64.86 & 85.97 & 107.04 & 121.09 \\
\hline 33 & 12.72 & 60.91 & 83.00 & 103.08 & 115.13 \\
\hline 37 & 10.78 & 54.96 & 75.06 & 97.12 & 111.18 \\
\hline 40 & 10.84 & 55.03 & 74.11 & 93.17 & 107.23 \\
\hline 43 & 12.92 & 51.15 & 73.16 & 91.23 & 103.28 \\
\hline 47 & 11.65 & 51.07 & 71.22 & 87.29 & 99.33 \\
\hline 50 & 11.58 & 45.26 & 69.28 & 83.34 & 97.39 \\
\hline 53 & 11.51 & 45.19 & 67.34 & 81.40 & 93.44 \\
\hline 57 & 11.44 & 43.31 & 65.40 & 79.45 & 91.50 \\
\hline 60 & 11.31 & 41.44 & 61.52 & 75.50 & 87.56 \\
\hline 63 & 11.24 & 41.37 & 61.54 & 73.56 & 85.61 \\
\hline 67 & 11.20 & 39.49 & 59.63 & 71.62 & 81.72 \\
\hline 70 & 11.14 & 37.62 & 59.59 & 69.68 & 81.66 \\
\hline
\end{tabular}




\begin{tabular}{|c|c|c|c|c|c|}
\hline 73 & 11.14 & 37.55 & 55.70 & 67.74 & 79.78 \\
\hline 77 & 11.14 & 37.75 & 53.82 & 67.80 & 78.87 \\
\hline 80 & 11.14 & 37.69 & 53.76 & 65.87 & 77.92 \\
\hline
\end{tabular}

For more accurate magnetoviscous behavior, we also collect the experimental data obtained by Nowak et al. [29], see table 3. The author chose a commercial biocompatible ferrofluid (FluidMAG DX) which is commonly used for preclinical studies of biomedical applications of ferrofluids. The concentration of the magnetic particles is stated as $25 \mathrm{mg} / \mathrm{ml}$. Measurements were carried out with an Anton Paar Physica MCR 301 rheometer with cone-plate geometry.

Table 3. Biocompatible ferrofluid viscosity at different magnetic flux density, Nowak et al. [29].

\begin{tabular}{|c|c|c|c|}
\hline \multirow{2}{*}{ Shear rate $\dot{\gamma}\left(\mathrm{s}^{-1}\right)$} & \multicolumn{3}{|c|}{ Experimental viscosity $\eta(\mathrm{mPa} \cdot \mathrm{s})$} \\
\hline & $\mathrm{B}_{\mathrm{o}}=10 \mathrm{kA} / \mathrm{m}$ & $\mathrm{B}_{\mathrm{o}}=20 \mathrm{kA} / \mathrm{m}$ & $\mathrm{B}_{\mathrm{o}}=30 \mathrm{kA} / \mathrm{m}$ \\
\hline 0.2 & 4.02 & 9.45 & \\
\hline 0.4 & 3.00 & 6.40 & \\
\hline 0.6 & 2.56 & 5.20 & \\
\hline 0.8 & 2.20 & 4.42 & \\
\hline 1.0 & 2.00 & 3.86 & 9.64 \\
\hline 2.0 & 1.50 & 3.00 & 6.50 \\
\hline 3.0 & 1.32 & 2.69 & 5.41 \\
\hline 4.0 & 1.33 & 2.54 & 4.80 \\
\hline 5.0 & 1.28 & 2.53 & 4.60 \\
\hline 6.0 & 1.27 & 2.37 & 4.28 \\
\hline 7.0 & 1.31 & 2.40 & 4.20 \\
\hline 8.0 & 1.21 & 2.40 & 4.10 \\
\hline
\end{tabular}

Different directions of the magnetic field also have a huge influence on magnetoviscous behavior. Linke and Odenbach [30] concluded that the highest magnetoviscous effect has been obtained in the magnetic field perpendicular to the flow field, table 4. In both low and high shear rate studies, the lowest magnetoviscous effect has been observed in a parallel orientation to the flow because the elongated microstructure oriented in the direction of the flow offers less hindrance to the flow. 
Table 4. $\mathrm{Fe}_{3} \mathrm{O}_{4}$ ferrofluid viscosity at different magnetic flux density, Linke et al. [30].

\begin{tabular}{|c|c|c|c|}
\hline \multirow{2}{*}{ Shear rate $\left.\dot{x} \mathrm{~s}^{-1}\right)$} & \multicolumn{3}{|c|}{ Experimental viscosity $\eta(\mathrm{mPa} \cdot \mathrm{s})$} \\
\cline { 2 - 4 } & $\mathrm{B}_{\mathrm{O}}=10 \mathrm{kA} / \mathrm{m}$ & $\mathrm{B}_{\mathrm{O}}=15 \mathrm{kA} / \mathrm{m}$ & $\mathrm{B}_{\mathrm{O}}=20 \mathrm{kA} / \mathrm{m}$ \\
\hline 1 & 102 & 120 & 130 \\
\hline 2 & 67 & 78 & 87 \\
\hline 3 & 55 & 63 & 63 \\
\hline 4 & 49 & 56 & 59 \\
\hline 5 & 45 & 52 & 56 \\
\hline 6 & 43 & 50 & 54 \\
\hline 7 & 42 & 48 & 52 \\
\hline 8 & 40 & 47 & \\
\hline
\end{tabular}

\section{CONSTITUTIVE EQUATION AND ELASTIC CHARACTERISTICS}

The flow behavior of MS can be described by the flow curve which is usually presented as a function of the form $\eta=f(\dot{\gamma})$. Most viscoelastic models are very simple and unable for the behavior description of the dilute polymer solutions. At present, the experimental measurements of the steady shear flow were fitted using Giesekus model. This model is suitable for polymer melts, dilute, semi-dilute and concentrated polymer solutions. The differential form of this model is given by, [1]:

$$
\begin{aligned}
& \underline{\underline{s}}=\underline{\underline{s}} s+\underline{\underline{s}} p \\
& \stackrel{s}{s}=2 \eta_{s} \stackrel{d}{=} \\
& \quad \stackrel{s}{=}+\lambda \stackrel{\nabla}{s}_{p}+\alpha \frac{\lambda 1}{\eta_{p}}\left(\stackrel{s}{s}_{p} \cdot \stackrel{s}{s}_{p}\right)=2 \eta_{p} \stackrel{d}{=}
\end{aligned}
$$

This model is written as a superposition of solvent and polymer contributions $s_{=s}$ and $s_{=p}$, to the stress tensor. The parameters $\eta_{s}$ and $\eta_{p}$ are the solvent and polymer contributions to the Newtonian or zero-shear-rate viscosity $\eta_{0}$ and $\alpha$ denotes the Giesekus mobility factor. The parameters $\eta_{0}$ and $\lambda_{2}$ are given in terms of $\eta_{s}$, and $\eta_{p}$ as:

$$
\eta_{\mathrm{o}}=\eta_{\mathrm{s}}+\eta_{\mathrm{p}} ; \quad \lambda_{2}=\lambda_{1} \frac{\eta_{\mathrm{s}}}{\eta_{\mathrm{o}}}
$$


The inclusion of : ${\stackrel{s}{s}, s_{p}}_{p}$ ) term in equation 26 gives a viscosity function that is more realistic than any other model. Giesekus model yields the following material functions (apparent viscosity $\eta$, first and the second normal stresses $\Psi_{1}$ and $\Psi_{2}$ ):

$$
\begin{aligned}
& \qquad \dot{\gamma})=\eta_{\mathrm{o}}\left\{\frac{\lambda_{2}}{\lambda_{1}}+\left(1-\frac{\lambda_{2}}{\lambda_{1}}\right) \frac{(1-f)^{2}}{1+(1-2 \alpha) f}\right\}, \\
& \Psi(\dot{\gamma})=2 \eta_{\mathrm{o}}\left(\lambda_{1}-\lambda_{2}\right)\left\{\frac{f(1-\alpha f)}{\left(\lambda_{1} \dot{\gamma}\right)^{2} \alpha(1-f)}\right\}, \\
& \Psi \dot{\gamma} \dot{\gamma})=\eta_{\mathrm{o}}\left(\lambda_{1}-\lambda_{2}\right)\left\{\frac{-f}{\left(\lambda_{1} \dot{\gamma}\right)}\right\},
\end{aligned}
$$

where

$$
f=\frac{1-\chi}{1+(1-2 \alpha) \chi}
$$

and

$$
\chi^{2}=\frac{\sqrt{1+16 \alpha(1-\alpha)\left(\lambda_{1} \dot{\gamma}\right)^{2}}-1}{8 \alpha\left(1-\alpha\left(\lambda_{1} \dot{\gamma}\right)^{2}\right.}
$$

$\Psi_{1}$ and $\Psi_{2}$ are not taken into account here because of they can be measured only in un-symmetric regions such as eccentric cylinders or cone and plate rheometers. This model is characterized by four parameters $\eta_{0}$, $\lambda_{1}, \lambda_{2}$ and $\alpha$, which can be treated as the model's parameters. Limiting cases of the Giesekus model include the Newtonian fluid ( $\alpha=\lambda_{1}=\lambda_{2}=0$ ), the upper-convected Maxwell fluid ( $\alpha=\lambda_{2}=0$ ) and the Oldroyd-B fluid $(\alpha=0)$. However, by adding a small retardation term (e.g., $\lambda_{2} / \lambda_{1}=10^{-3}$ ), the magnitude of the shear stress is always increasing with increasing shear rate. In general we must require $0<\alpha<1$ for realistic shear thinning and $\alpha>1$ for shear thickening fluids bearing in mind that $\lambda_{1}>\lambda_{2}$.

\section{RESULTS AND DISCUSSION}

\section{Magnetic suspensions flow curves}

In this section, fitting results of the experimental data given in [27-30] are overviewed to give a coherent picture about the rheological behaviors of the considered suspensions. Four sets of experimental data were used in order to describe their rheological behavior, see tables 1, 2,3 and 4. A good fit for the experimental flow curves can be obtained starting from the understanding the effects of the Giesekus parameters on the flow curve under consideration and finding the proper values for these parameters. Then by inserting the proper values in Giesekus model explained above we can draw a theoretical curves. The proper parameter values must be able to the theoretical curve to catch the experimental points. The suitable Giesekus parameters required for the best fit of the four sets of experimental data are listed in table 5.

Table 5. Giesekus parameters for used ferrofluid suspensions at different magnetic flux density.

\section{Author(s)}

Magnetic Flux

\section{Giesekus Model Parameters}




\begin{tabular}{|c|c|c|c|c|c|}
\hline & $\mathrm{B}_{\mathrm{O}}$ & $\eta_{d}$ (Pa.s) & $\lambda 1 s)$ & $\lambda_{2} / \lambda_{1}$ & $\alpha$ \\
\hline \multirow{3}{*}{ Vshivkov et al. [27] } & $0 \mathrm{kA} / \mathrm{m}$ & 0.700 & 0.70 & 0.060 & 0.2 \\
\hline & $280 \mathrm{kA} / \mathrm{m}$ & 1.800 & 0.50 & 0.060 & 0.2 \\
\hline & $290 \mathrm{kA} / \mathrm{m}$ & 5.000 & 0.70 & 0.045 & 0.2 \\
\hline \multirow{5}{*}{ Dhumal et al. [28] } & $0.0 \mathrm{~T}$ & 0.090 & 0.70 & 0.060 & 0.2 \\
\hline & $0.1 \mathrm{~T}$ & 0.350 & 0.46 & 0.060 & 0.2 \\
\hline & $0.2 \mathrm{~T}$ & 0.420 & 0.35 & 0.065 & 0.2 \\
\hline & $0.3 \mathrm{~T}$ & 0.500 & 0.35 & 0.068 & 0.2 \\
\hline & $0.4 \mathrm{~T}$ & 0.620 & 0.39 & 0.074 & 0.2 \\
\hline \multirow{3}{*}{ Nowak et al. [29] } & $10 \mathrm{kA} / \mathrm{m}$ & 0.005 & 2.80 & 0.250 & 0.8 \\
\hline & $20 \mathrm{kA} / \mathrm{m}$ & 0.012 & 3.60 & 0.180 & 0.8 \\
\hline & $30 \mathrm{kA} / \mathrm{m}$ & 0.030 & 2.80 & 0.120 & 0.8 \\
\hline \multirow{3}{*}{ Linke et al. [30] } & $10 \mathrm{kA} / \mathrm{m}$ & 0.210 & 1.60 & 0.150 & 0.8 \\
\hline & $15 \mathrm{kA} / \mathrm{m}$ & 0.240 & 1.60 & 0.150 & 0.8 \\
\hline & $20 \mathrm{kA} / \mathrm{m}$ & 0.270 & 1.60 & 0.150 & 0.8 \\
\hline
\end{tabular}

Figures 5 show that, by choosing a suitable model's parameters, Giesekus curve and the experimental points overlap each other or appear to have only a small deviation. This indicates that, the Giesekus model fit is reasonable for the viscosity of all MS. Tables 6 , shows the most suitable parameter values of equation 28 .

Figure 5 a shows the shear dependence of viscosity for $\mathrm{Fe}_{2.8} \mathrm{O}_{4}$ suspensions in the presence and in the absence of a magnetic field. It is obvious that this suspension has shear thinning behavior, this also reported for other ferrofluids such as $\mathrm{Fe}_{3} \mathrm{O}_{4}$ and MAG DX in different base fluids, figures. $5 b, 5 c$ and $5 d$. The studied suspensions have a non-Newtonian fluid behaviors, where the viscosity is decreasing when the shear rate increases. As seen from the figures, samples exhibit shear thinning over the whole shear rate range investigated. When the ferrofluid exposed to the magnetic field, the magnetic particles tend to arrange themselves along the direction of the magnetic field (in this study, perpendicular to flow direction) and form new structures with higher resistance under the magnetic field. By increasing the field intensity, the interaction between the magnetic particles and their arrangement become stronger. Therefore, the viscosity of ferrofluid increases under an application of the field. By increasing shear rates, the viscosity drops significantly and its profile changes to one exhibiting three discrete regions as following:

- Low shear rates, in which the velocity profile consists of a shear thinning. These situations indicate the breaking of the initial structures of magnetic particles.

- Intermediate shear rates, where the suspension viscosity continues to decrease and the particles chains orient along the flow direction during deformation. 
- High shear rates region, where the shear stress is high enough to destroy the suspension domains into individual particle chains and orient these chains along the flow direction. Therefore, the viscosity tends to become a constant value.
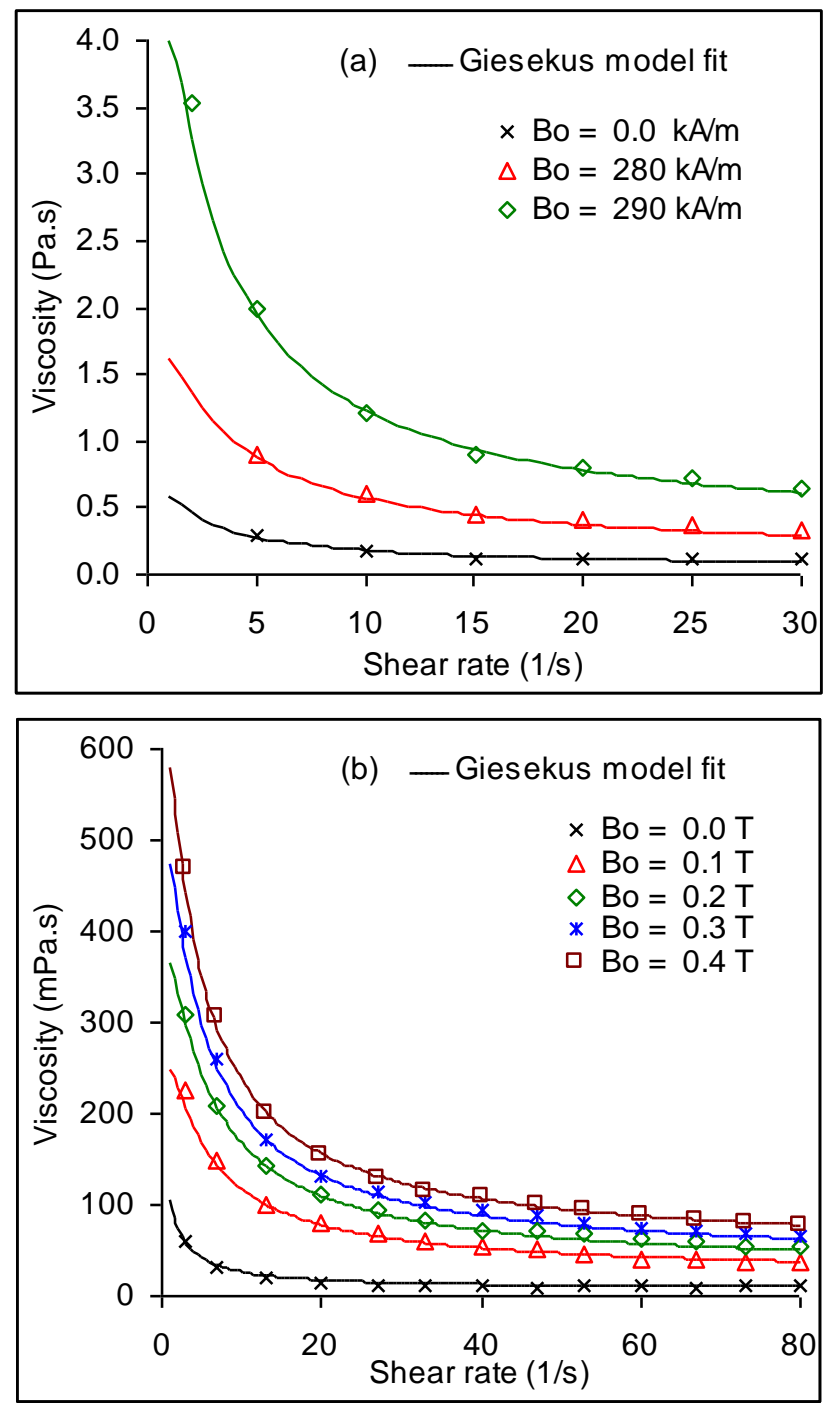

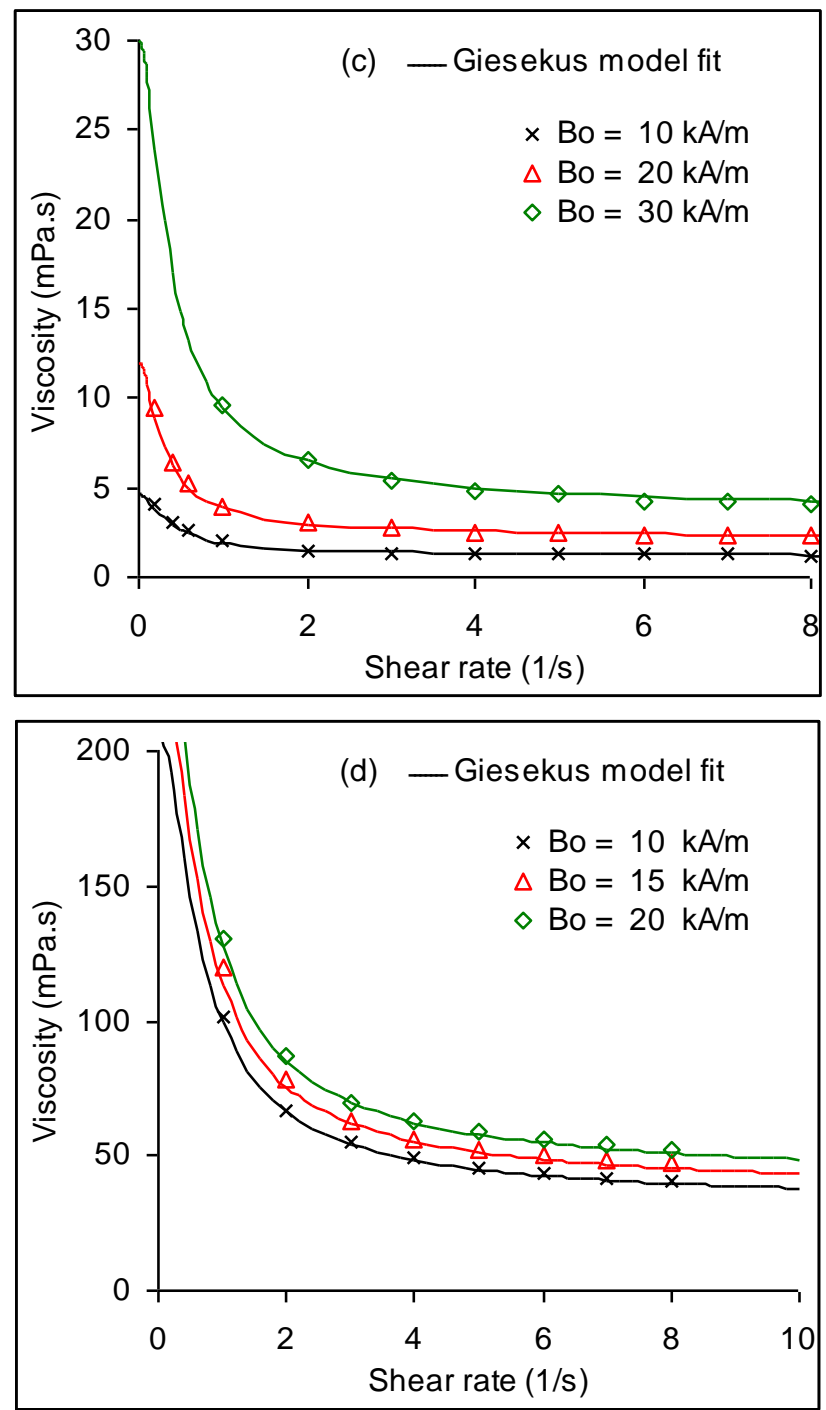

Fig 5: Viscosity of ferrofluids under different magnetic flux densities where lines represent Giesehus model fits and marks represent experimental data of (a) Vshivkov et al. [27], (b) Dhumal et al. [28], (c)Nowak et al. [29] and (d) Linke et al. [30].

\section{Magnetoviscous effect and the suggested correlation}

During analysis of the viscosity data, we must take into account that it is affected by shear rate and the applied magnetic field. As we have seen above, the viscosity of all samples decreases with increasing shear rate and increases with increasing the applied magnetic field. To study the effect of magnetic field on the rheological behavior of MS, data measured by Dhumal et al. [28] are considered. The range of shear rate is sufficient to cover the regions in which the viscosities at high shear rates arise to some constant values. In the range of low and intermediate magnetic fields, the viscosity is a function of magnetic field. Similar dependence were obtained from that data measured by Vshivkov et al. [27], Nowak et al. [29] and Linke et al. [30].

The relationship between magnetic field and viscosity of iron oxide $\mathrm{Fe}_{3} \mathrm{O}_{4}$ suspensions at constant shear rates is shown in figure 6a. It is clear that, when shear rate is zero, applying a constant magnetic field causes the formation of large chains. Then, by applying a small shear rate the particles will find a chance to move and rearrange to form small chains. However, at higher shear rate, the shear force can break the chains and the magnetoviscous effect is weakened. It also notices that for all different magnetic fields in figure $6 b$, the behavior of $\mathrm{Fe}_{3} \mathrm{O}_{4}$ suspension is shear thinning. The magnetic field leads to magnetization of magnetic 
particles and then they adhere to each other. Therefore, aggregated structures are formed and these structures increase the viscosity. In spite of applying the shear rate, these structures don't break; in other words the shear force is not too strong to break down these structures. With more increasing of shear rate the applied shear stress overcome to the strength of these structures and then the viscosity decreases gradually.

For practical engineering, it is useful to get a simple equation describing the combined effect of magnetic field and shear rate on MS. The magnetoviscous effect shown in figure 6a reveals that formation of new structures is occurring as a function of the magnetic field. As we have seen in the previous sections, Giesekus model accurately predicts the viscosity as a function of shear rate equation 28. Referring to my previous works [3133] we can relate the measured viscosity to shear rate and magnetic flux density. Therefore, we introduce a new proposed correlation which predicts the viscosity of $\mathrm{Fe}_{3} \mathrm{O}_{4}$ ferrofluid in the form:

$$
\left.\left.\eta \dot{\gamma}, \mathrm{B}_{\mathrm{O}}\right)=\left(19.2 \mathrm{~B}_{\mathrm{O}}^{3}-13.5 \mathrm{~B}_{\mathrm{O}}^{2}+3.6 \mathrm{~B}_{\mathrm{O}}+0.1\right) \eta \dot{\gamma}\right) .
$$

Since Giesekus model accurately predicts the viscosity as a function of shear rate, the term $\left(19.2 \mathrm{~B}_{\mathrm{O}}^{3}-13.5 \mathrm{~B}_{\mathrm{O}}^{2}+3.6 \mathrm{~B}_{\mathrm{O}}+0.1\right)$ is equivalent to $\eta_{\mathrm{o}}$ in equation 28 and the term $\frac{1}{\gamma} \dot{\gamma}$ ) is given by:

$$
\bar{\eta}=\frac{\lambda_{2}}{\lambda_{1}}+\left(1-\frac{\lambda_{2}}{\lambda_{1}}\right) \frac{(1-f)^{2}}{1+(1-2 \alpha) f}
$$

by correlating the experimental results using Mathematica program, Giesekus parameters $\lambda_{1}, \lambda_{2}$ and $\alpha$ as a functions of magnetic field strength are listed in table 6 . In order to evaluate the accuracy of this correlation, comparison between the proposed model, equation 33, and the experimental viscosity data at different magnetic field strengths were plotted in figure $6 \mathrm{~b}$. In most measured data, the curve of correlation and the experimental points overlap each other or appear to have slight deviation. This indicates that, the suggested correlation has a suitable accuracy and equation 33 is reasonable for the viscosity of $\mathrm{Fe}_{3} \mathrm{O}_{4}$ ferrofluid. Moreover, the results of the predicted model are in excellent correspondence with those of the experimental data in the most range of magnetic flux density. The combined effects of shear rate and magnetic field on MS viscosity can be shown in figure 7 (in 3 dimensional form). 

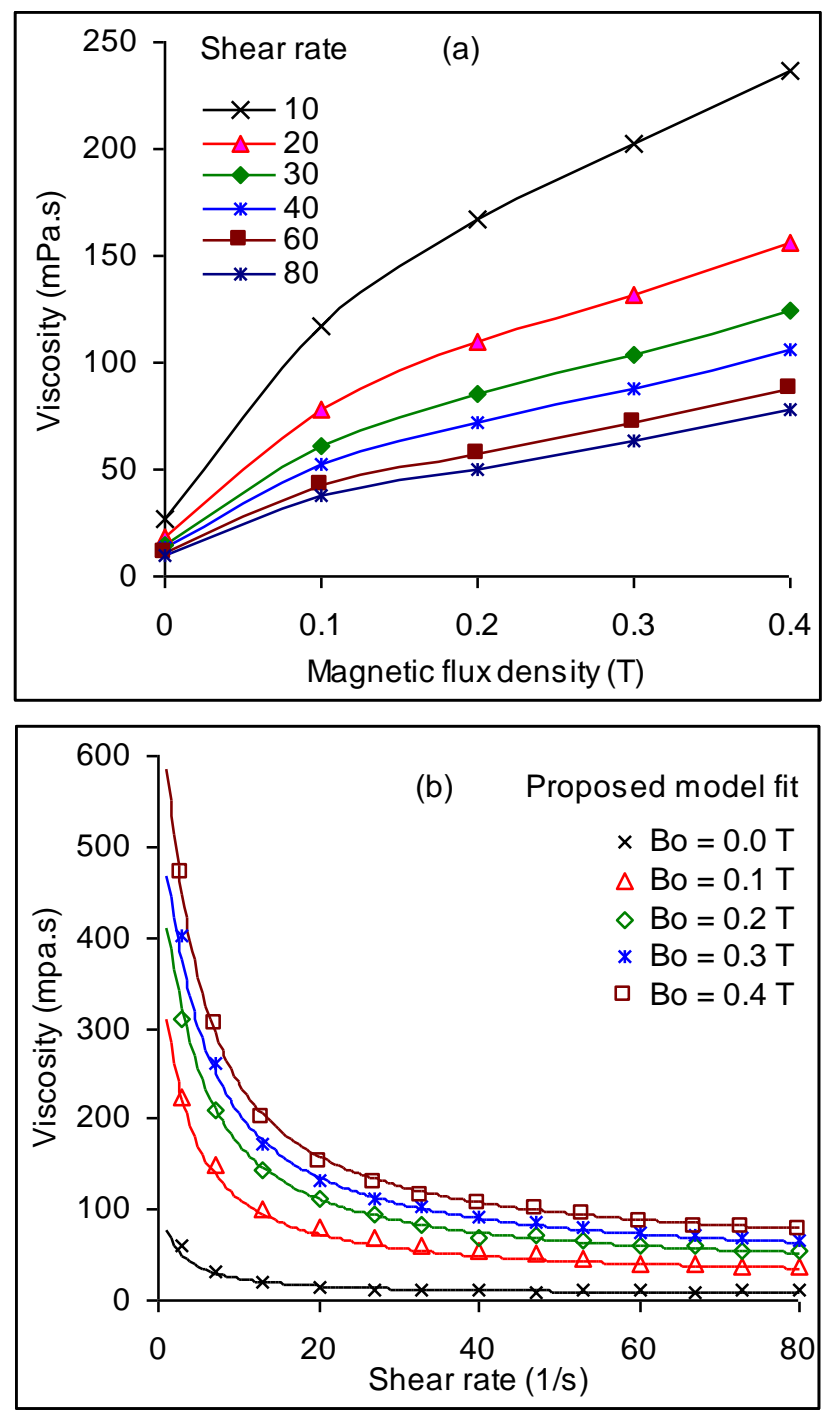

Fig 6: (a) Magnetoviscous effect of $\mathrm{Fe}_{3} \mathrm{O}_{4}$ ferrofluid under different magnetic flux density, (b) Comparison between the proposed model and the experimental viscosity data at different magnetic field strengths.

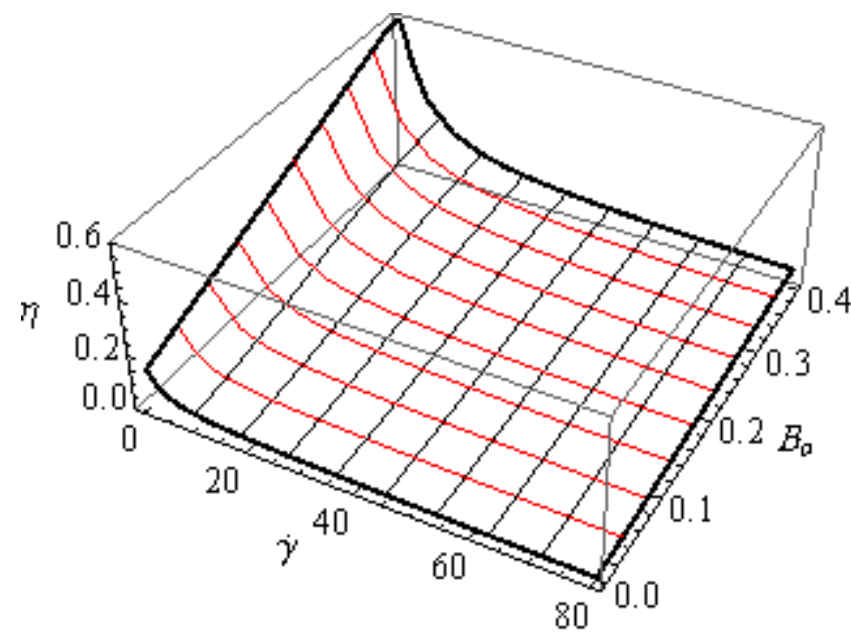

Fig 7: The combined effects of shear rate and magnetic field on $\mathrm{Fe}_{3} \mathrm{O}_{4}$ ferrofluid suspension in $3 \mathrm{D}$. 
Table 6. Suggested correlation parameters for $\mathrm{Fe}_{3} \mathrm{O}_{4}$ ferrofluid as functions of magnetic flux density.

\begin{tabular}{|c|c|c|c|c|}
\hline \multirow{2}{*}{$\mathrm{B}_{\mathrm{O}}$} & \multicolumn{3}{|c|}{ Giesekus model parameters } \\
\cline { 2 - 5 } & $\eta_{\mathrm{d}}$ (Pa.s) & $\lambda 1 \mathrm{~s})$ & $\lambda_{2} / \lambda_{1}$ & $\alpha$ \\
\hline OT $-0.4 \mathrm{~T}$ & $19.2 \mathrm{~B}_{\mathrm{O}}^{3}-13.5 \mathrm{~B}_{\mathrm{O}}^{2}+3.6 \mathrm{~B}_{\mathrm{O}}+0.1$ & $4.5 \mathrm{~B}_{\mathrm{O}}^{2}-2.6 \mathrm{~B}_{\mathrm{O}}+0.7$ & $0.7 \mathrm{~B}_{\mathrm{O}}^{2}+0.01 \mathrm{~B}_{\mathrm{O}}+0.1$ & 0.2 \\
\hline
\end{tabular}

\section{CONCLUSIONS}

The main objective of the theoretical solution is to examine in detail the basic MHD flow of a magnetic suspension between two concentric cylinders. The momentum equation and the equation of state that characterize the suspension are solved in exact manner considering the Lorentz force to account the resistance offered by the magnetic field. The obtained velocity profiles possess modified Bessel functions of first and second kind. The effects of various parameters on the flow characteristics such as viscoelasticity, magnetic parameters have been studied and discussed graphically. Therefore, we conclude the following remarks:

- An increase in magnetic parameter $m$ reduces the velocity profile monotonically due to the effect of magnetic force against the flow direction (magnetoviscous effect).

- $\quad$ An increase in the parameter $\beta$ yields an effect opposite to that of the magnetic field.

To confirm these conclusions, some experimental results measured in more than one source have been studied and analyzed. The flow curves of all selected MS under different magnetic fields show that the viscosity decreases with increasing the shear rate. Therefore, Giesekus model is used to describe the viscoelasticity nature of the studied MS. The curves show also that this model fits the experimental data very well. Therefore, Giesekus model is currently the adequate one describing the rheological properties of MS with or without applied magnetic field. By experimental measurement and theoretical analysis, the following conclusions can be addressed:

- The MS demonstrated the shear thinning behavior.

- Giesekus model could describe the magnetic suspension, whenever the suspension is subjected to applied magnetic field or not.

- Magnetic particles exist as aggregates in the suspension and these aggregates further formed chaining structure under applied magnetic field.

- The mechanical toque induced by the shear flow would divert the chain-like aggregates formed by the field.

- The external magnetic field gives rise to a magnetic torque to contrast action the mechanical torque.

- When the shear rate was large enough such that the aggregate structures had been broken, the viscosity would not change any more with the increasing magnetic field. 
- A general constitutive equation was proposed to calculate the viscosity of MS subjected to applied magnetic field.

\section{REFERENCES}

[1] Bird, R.B., Armstrong, R.C. and Hassager, O. Dynamics of polymeric liquids. Wiley-Interscience, New York (1987).

[2] Dery, J.P., Borra, E.F. and Ritcey, A.M. Ethylene glycol based ferrofluid for the fabrication of magnetically deformable liquid mirrors. Chemistry of Materials, 20 (2008) 6420-2426.

[3] Sansom, C.L., Jones, P., Dorey, R.A., Beck, C., Stanhope-Bosumpim, A. and Peterson, J. Synthesis and characterization of $\mathrm{Mn}_{0.5} \mathrm{Zn}_{0.5} \mathrm{Fe}_{2} \mathrm{O}_{4}$ and $\mathrm{Fe} 3 \mathrm{O} 4$ nanoparticle ferrofluids for thermo-electric conversion. Journal Magnetism and Magnetic Materials, 335 (2013) 159-162.

[4] Atif, S.M., Hussain, S. and Sagheer, M. Numerical study of MHD micropolar carreau nanofluid in the presence of induced magnetic field. AIP Adv, 8 (2018) 1-20.

[5] Rinaldi, C., Chaves, A., Elborai, S., He, X. and Zahn, M. Magnetic fluid rheology and flows. Curr Opin Colloid Interface Sci, 10 (2005) 141-157.

[6] Pop, L.M., Hilljegerdes, J., Odenbach, S. and Wiedenmann, A. The microstructure of ferrofluids and their rheological properties. Appl Org Chem, 18 (2004) 523-528.

[7] Odenbach, S. Recent progress in magnetic fluid research. J Phys Condens Matter, 16 (2004) 11351150.

[8] Gan Jia Gui, N., Stanley, C., Nguyen, N.T. and Rosengarten, G. Ferrofluids for heat transfer enhancement under an external magnetic field. Int J Heat Mass Trans, 123 (2018) 110-121.

[9] Kimura, T. Study on the effect of magnetic fields on polymeric materials and its application. Polym J, 35 (2003) 823-843.

[10] Vshivkov, S.A. and Rusinova, E. Effect of magnetic field on the rheological properties of poly(ethylene glycol) and poly(dimethylsiloxane) mixtures with aerosil and iron nanoparticles. Poly Sci A, 59 (2017) 764-771.

[11] Ghasemi, E., Mirhabibi, A. and Edrissi, M. Synthesis and rheological properties of an iron oxide ferrofluid. J Magn Magn Mater, 320 (2008) 2635-2639.

[12] Hong, R.Y., Ren, Z.Q., Han, Y.P., Li HZ, Zheng, Y. and Ding, J. Rheological properties of water-based $\mathrm{Fe}_{3} \mathrm{O}_{4}$ ferrofluids. Chem Eng Scie, 62 (2007) 5912-5924.

[13] Odenbach, S. and Stork, H. Shear dependence of field-induced contributions to the viscosity of magnetic fluids at low shear rates. J Magn Magn Mater, 183 (1998) 188-194.

[14] Yu Zubarev, A., Fleischer, J. and Odenbach, S. Towards a theory of dynamical properties of polydisperse magnetic fluids: Effect of chain-like aggregates. Physica A, 358 (2005) 475-491.

[15] Skumryev, V., Stoyanov, S., Zhang, Y., Hadjipanayis, G., Givord, D. and Nogues, J. Beating the superparamagnetic limit with exchange bias. Nature, 423 (2003) 850-853.

[16] De Gans, B.J., Duin, N.J., Van den Ende, D. and Mellema J. The influence of particle size on the magnetorheological properties of an inverse ferrofluid. J Chem Phys, 113 (2000) 2032-2042. 
[17] Wang, J.M., Kang, J.F., Zhang, Y.J. and Huang, X.J. Viscosity monitoring and control on oil-film bearing lubrication with ferrofluids. Tribol Int, 75 (2014) 61-68.

[18] Shchukin, D.G., Radtchenko, I.L. and Sukhorukov, G.B. Micron-scale hollow poly-electrolyte capsules with nanosized magnetic $\mathrm{Fe}_{3} \mathrm{O}_{4}$ inside. Mater Lett, 57 (2003) 1743-1747.

[19] Chiriac, H. and Stoian, G. Influence of the particles size and size distribution on the magnetorheological fluids properties. IEEE Trans Magn, 45 (2009) 4049-4055.

[20] Berger, P., Adelman, N.B., Beckman, K.J., Campbell, D.J., Ellis, A.B. and Lisensky, G.C. Preparation and properties of an aqueous ferrofluid. J Chem Educ, 76 (1999) 943-948.

[21] Love, J.C., Urbach, A.R., Prentiss, M.G. and Whitesides, G.M. Three dimensional self-assembly of metallic rods with submicron diameters using magnetic interactions. J Am Chem Soc, 125 (2003) 12696-12697.

[22] Shah, K., Oh, J.S., Choi, S.B. and Upadhyay, R. Plate-like iron particles based bidisperse magnetorheological fluid. J Appl Phys, 114 (2013) 213904.

[23] Dai, J., Yang, M., Li, X., Liu, H. and Tong, X. Magnetic field sensor based on magnetic fluid clad etched fiber Bragg grating. Opt Fiber Tech, 17 (2011) 210-213.

[24] Odenbach, S. and Colloids, J.S. Colloids and surfaces A: physicochemical and engineering aspects. Physicochem. Eng Aspects, 217 (2003) 171.

[25] Sheparovych, R., Sahoo, Y., Motornov, M., Wang, S., Luo, H., Prasad, P.N., Sokolov, I. and Minko, S. Polyelectrolyte stabilized nanowires from $\mathrm{Fe}_{3} \mathrm{O}_{4}$ nanoparticles via magnetic field induced self assembly. Chemistry of Materials, 18 (2006) 591-593.

[26] Ding, X., Sun, Z., Zhang, W., Peng, Y., Chan, A.S.C. and Li, P. Characterization of Fe3O4/poly(styrene-co$\mathrm{N}$-isopropylacrylamide) magnetic particles with temperature sensitivity. Colloid Polym Sci, 278 (2000) 459-463.

[27] Vshivkov, S.A., Rusinova, E.V. and Galyas, A.G. Effect of a magnetic field on the rheological properties of iron oxide-water-glycerol system. Rheol Acta, 55 (2016) 155-161.

[28] Dhumal, J., Bandgar, S., Zipare, K. and Shahane, G. Fe3O4 ferrofluid nanoparticles: synthesis and rheological behavior. Int J of Materials Chem and Phys, 1 (2015) 141-145.

[29] Nowak, J. and Odenbach, S. Magnetoviscous effect in a biocompatible ferrofluid. IEEE Trans on Magnetics, 49 (2013) 208-212.

[30] Linke, J.M. and Odenbach, S. Anisotropy of the magnetoviscous effect in a ferrofluid with weakly interacting magnetite nanoparticles. J Phys Condens Matter, 27 (2015) 176001.

[31] Hamza, S.E.E. A comparison of rheological models and experimental data of metallocene linear low density polyethylene solutions as a function of temperature and concentration. J Adv Phys, 12 (2016) 43224339.

[32] Hamza, S.E.E. Modelling the effect of concentration on non-Newtonian apparent viscosity of an aqueous polyacrylamide solution. Global J Phys, 5 (2016) 505-517.

[33] Hamza, S.E.E. MHD flow of cellulose derivatives and dilute suspensions rheology of its nanocrystals. American Journal of Fluid Dynamics, 7 (2017) 23-40. 


\section{Author' biography with Photo}

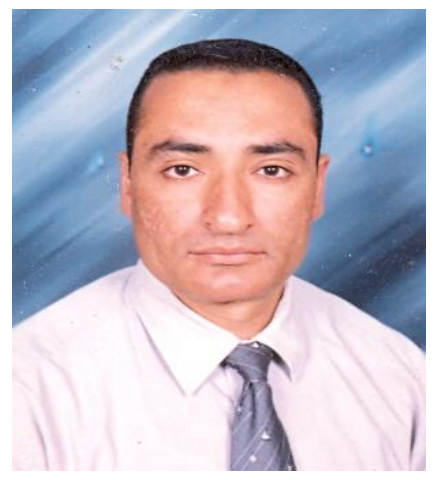

Ass. Prof. S.E.E. Hamza born in Hasanea, Touk, Kalubea, Egypt on $6^{\text {th }}$ January 1968. Since 1991 working at Physics Deparment, Faculty of Science, Benha, Egypt. Obtained his Ph. D. degree in theoretical physics in 2003 from Benha University under the able guidance of Prof. M. Zidan, Prof. A. Abu-El Hassan and Prof. N.M. El Nagar. He is working as Assistant Professor, Faculty of Science, Benha, Egypt in Physics department. Two students, one obtained M.Sc. degree and the other obtained Ph.D. degree under his Guidence. He is guiding one student for M. Sc. The topics of interest are Fluid Dynamics Nonlinear differential equations, BioPhysics. 